Submitted to: 7th Symposium on Electron Beam Ion Sources (EBIS-7)

Gelnhausen, Germany, 9/1-5/97

7 th International Conference on Ion Sources (ICIS 197)

Taormina, Italy, 9/7-13/97 CONF-970958--

BNL -64732

REVIEW OF ELECTRON BEAM MACROINSTABILITIES AND OTHER EBIS

RELATED STABILITY AND ISSUES*

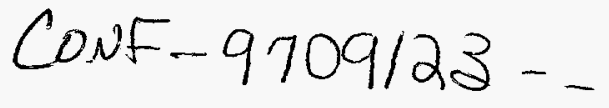

Ady Hershcovitch

AGS Department, Brookhaven National Laboratory

Upton, N.Y. 11973, U.S.A.

PEOPNDQR

NoY 12 1997

OST1

Plasma magnetohydrodynamics and macro-instability theories are briefly reviewed. Although the configuration of any EBIS is inherently susceptible to a number of classical beam instabilities, the small radial dimension of an EBIS plasma prevents modes from occurring in EBIS traps with low beam compression due to physical limitation. In EBIS devices with high electron beam compression, where the potential for beam instabilities is great, the radial dimension is smaller than the Debye length, which renders any plasma theory invalid. However, a RHIC EBIS is expected to have a diameter which is much larger than the Debye length. Hence, it may be the first EBIS, in which the various plasma theories could be valid. For this and future devices like it, a framework is established to analyze and offer remedies plasma instabilities in EBIS.

\title{
MASTER
}

*Work performed under the auspices of the U.S. Department of Energy. 


\section{I). Introduction}

Plasma MHD theory and examples of macro-instabilities are briefly reviewed. The main objective of this paper is to complement a paper on plasma kinetic theory and on beam micro-instabilities, which was presented at the Sixth International Symposium on Electron Beam Ion Sources and Their Applications three years ago.[1] An addendum on microinstabilities for that paper will be also included in this paper (to incorporate a better spatial model for an EBIS plasma).

The review of the fundamentals of plasma kinetic theory, which was presented at the Sixth International Symposium on Electron Beam Ion Sources and their Applications, was in response to a number of controversial papers on the stability of EBIS devices. One of the reasons for the controversy is the fact that, in many of the papers on this subject matter, plasma theories have been applied to analyze experimental devices without first exploring whether those theories are valid for the experimental parameters they analyze. Kinetic theory, macro-, and micro-instabilities have been no exception. Additionally, in a number of these papers no distinction is made with regards to the class of instabilities (including those resulting from interactions with the EBIS structure).

Poor performance in some EBIS devices, especially when coupled with observation of rf signal, was occasionally blamed on plasma instabilities. When trying to attribute anomalous behavior in an EBIS to various plasma instabilities, questions were raised as to whether there is a simpler explanation like misalignment. Or, is the generated rf noise due to excitation and/or amplification of electromagnetic waves in the electron beam and trap structure rather than plasma type instabilities. 


\section{DISCLAMMER}

Portions of this document may be illegible in electronic image products. Images are produced from the best available original document. 


\section{DISCLAIMER}

This report was prepared as an account of work sponsored by an agency of the United States Government. Neither the United States Government nor any agency thereof, nor any of their employees, make any warranty, express or implied, or assumes any legal liability or responsibility for the accuracy, completeness, or usefulness of any information, apparatus, product, or process disclosed, or represents that its use would not infringe privately owned rights. Reference herein to any specific commercial product, process, or service by trade name, trademark, manufacturer, or otherwise does not necessarily constitute or imply its endorsement, recommendation, or favoring by the United States Government or any agency thereof. The views and opinions of authors expressed herein do not necessarily state or reflect those of the United States Government or any agency thereof. 
An addendum to the kinetic theory review is also included in this paper. The purpose of that review was to provide EBIS researchers with a mathematical formalism and a generalized dispersion relation, which are applicable to EBIS configurations in cases where plasma kinetic theory is valid. The objective of the addendum is to further refine the formalism for a more accurate mathematical representation of the EBIS plasma.

Similarly, the review of macro-instabilities will end up with generalized dispersion relations, which are applicable to EBIS in cases where MHD theory is valid. The generalized dispersion relation could be further reduced for parameters of a given instability to yield a simplified dispersion relation. To obtain instability growth rates, dominant modes and stability criteria, the dispersion relation can be solved (although in most cases, numerical methods are needed). Since there are a variety of EBIS devices with different parameters, the dispersion relation for each EBIS will be somewhat different. It will, obviously, depend on the parameters inside the trap, but, a realistic model should also exhibit a strong dependence on the electron beam parameters and geometry outside the trap.

Finally, a warning! Even in cases where the various plasma theories are valid, simpler explanations should be pursued first rather than instabilities. A good example, which the author experienced, was on SuperEBIS, where a specrta centered at about $100 \mathrm{MHZ}$ was observed. At low to moderate electron beam currents $350 \mathrm{~mA} \leq$, the spectra shift to higher frequencies as the electron beam current increases. Those frequencies disappeared with proper transverse magnetic field adjustments, with very strong sensitivity to the coils close to the collector. Beam rotation resulting from a combination of beam displacement and rigid rotor motion can account for the observed phenomena. However, as is the case with many 
observed frequencies in plasmas, there is also an instability "to match" those frequencies. In this case, the resonant diocotron instability can also account for the observed phenomena, especially for the strong dependence on the coils before the collector, and the multitude of observed frequencies (although non-uniformities in the electron beam can also yield a spectrum of frequencies).

Beam rotation seems to be a more fundamental explanation (mathematically relying on $0^{\text {th }}$ order effect). It is simpler, it requires only that the electron beam be off axis. Unlike the instability which requires the existence of a sign change in the radial density gradient, which is a necessary condition for any diocotron instability.

\section{II). Addendum to Review of Electron Beam Micro-Instabilities and their Relevance to}

\section{EBIS Devices}

A review of the fundamentals of plasma kinetic theory was presented at the $6^{\text {th }}$ International Symposium on Electron Beam Ion Sources and their Applications.[1] That review was in response to the controversy regarding EBIS stability.

The purpose of that review was to provide EBIS researchers with a mathematical formalism and a generalized dispersion relation, which are applicable to EBIS configurations in cases where plasma kinetic theory is valid. The objective of this addendum is to further refine the model for a more accurate mathematical representation of the EBIS plasma. The generalized dispersion relation will be further reduced to a form comprising of functions which, for parameters of a given instability, can be expanded to yield a simplified dispersion relation. To obtain instability growth rates, dominant modes and stability criteria, the dispersion relation can be solved straightforward (although in some cases, numerical 
methods are needed). Due to the variety of EBIS devices and wide range in parameters, the dispersion relation for each EBIS will be somewhat different.

Slab geometry, which was used in that review, is fairly good description for some fusion devices. However, it is inappropriate for EBIS due to the very steep spatial gradients in the radial direction. The dispersion relation obtained in that review was

$$
\begin{gathered}
0=1-\sum_{j=i, e} \frac{\omega_{\mathrm{p}_{\mathrm{e}}}^{2}}{k^{2}} \int \mathrm{d} v\left\{\mathrm { i } R \left[k_{\mathrm{i}} \frac{\partial}{\partial v_{\|}}+\left(\omega-k_{\|} v_{\|}\right) \frac{1}{v_{\perp}} \frac{\partial}{\partial v_{\perp}}\right.\right. \\
\left.\left.-\frac{1}{\Omega_{j}}\left(k_{\dot{\theta}} \frac{\partial(r)}{\partial r}-k_{r} \frac{\partial}{\partial \theta}\right)\right]+\frac{1}{v_{\perp}} \frac{\partial}{\partial v_{\perp}}\right\} \text { foj }\left(r, v_{\perp}, v_{\|}\right), \\
R=\sum_{n=-\infty}^{\infty} \frac{\mathrm{i} J_{n}^{2}\left(k_{\perp} v_{\perp} / \Omega_{j}\right)}{\omega-k_{\mathrm{i}} v_{\|}-n \frac{\Omega_{j}}{2}\left\{1 \pm\left[1-\frac{2 \omega_{\mathrm{p}_{\mathrm{i}}}^{2}}{\Omega_{j}^{2}}(1-\phi)\right]^{1 / 2}\right\}}
\end{gathered}
$$

Same glossary as in reference 1 is used throughout this paper, except where noted. In equation $1, \phi$ is used to denote neutralization fraction (instead of $\mathrm{f}$ in reference 1). Also, cylindrical coordinated are used for configuration space in equation 1 , instead of Cartesian. The summation in equation 1 must be performed over all particle species. That includes the various ion charge states of the same ion species.

Spatially, Gaussian distribution is used to model the plasma radial profile. 


$$
\begin{gathered}
f \circ \mathrm{i}=\frac{1}{\pi^{3 / 2} v_{\mathrm{th}_{\mathrm{i}}}^{3}} \exp \left(-\frac{v_{\mathrm{l}}^{2}+v_{\perp}^{2}}{v_{\mathrm{th}_{\mathrm{i}}}^{2}}\right) \exp \left[-\frac{r^{2}}{R_{\mathrm{i}}^{2}}\right], \\
f \circ=\frac{1}{\pi^{3 / 2} v_{\mathrm{th}_{\mathrm{le}}} v_{\mathrm{th}_{\perp \mathrm{e}}}^{2}} \exp \left(-\frac{v_{\perp}^{2}}{v_{\mathrm{th}_{\perp \mathrm{c}}}^{2}}\right) \exp \left(-\frac{\left(v_{\|}-v_{o}\right)^{2}}{v_{\mathrm{th}_{\perp \mathrm{e}}}^{2}}\right) \exp \left[-\frac{r^{2}}{R_{\mathrm{e}}^{2}}\right],
\end{gathered}
$$

To obtain a dispersion relation with those distribution functions, equations 2 are substituted in equation 1. After some manipulations, a dispersion relation in term of $\mathrm{Z}$ functions is obtained, to adequately characterize the plasma in the trap

$$
\begin{aligned}
& \epsilon=0=1+2 \frac{\omega_{\mathrm{pe}}^{2}}{k^{2} v_{\text {the }}^{2}}\left[1+\frac{\omega-\omega_{* e}}{k_{\|} v_{\text {the }}} \sum_{n=-\infty}^{\infty} \exp \left(-b_{e}\right) I_{n}\left(b_{e}\right)\right. \\
& \times Z\left(\frac{\omega-k_{1} v_{o}+n \Omega_{e}\left\{1 \pm\left[1-\frac{2 \omega_{\mathrm{pe}}^{2}}{\Omega_{e}^{2}}(1-\phi)\right]^{1 / 2}\right\}}{k_{\mathrm{l}} v_{\text {the }}}\right)+\sum_{\mathrm{j}} 2 \frac{\omega_{\mathrm{pij}}^{2}}{k^{2} v_{\mathrm{thi}}^{2}} \\
& \mathrm{x} \sum_{n=-\infty}^{\infty} \frac{\omega-\omega_{\mathrm{ijj}}}{k_{\|} v_{\mathrm{thi}}} \mathrm{Z}\left(\frac{\omega+n \Omega_{\mathrm{ij}}\left\{1 \pm\left[1+\frac{2 \omega_{\mathrm{pij}}^{2}}{\Omega_{\mathrm{e}}^{2}}\left(\frac{1-\phi}{\phi}\right)\right]^{1 / 2}\right\}}{k_{\mathrm{l}} v_{\mathrm{thij}}}\right) I_{n}\left(b_{i j}\right) \exp \left(-b_{i j}\right) \\
& \text { where } \\
& b_{j}=\frac{1}{2}\left(k_{\perp} v_{t} / \Omega_{j}\right)^{2}, \text { and } \omega_{* j}=-\frac{1}{2}\left(k_{\perp} v_{r j}^{2} / \Omega_{j} R\right)
\end{aligned}
$$


Again, $\mathrm{j}$ refers to a summation over all ion species and charge states. Depending on the mode and plasma parameters, equation 3 can be solved using numerical, and where possible analytical, forms of the well-known $\mathrm{Z}$ function.[2] Outside the trap, the electron beam is unneutralized, hence no ion terms. At the gate of a trap, there should be other terms which account for ion gradients in the $\mathrm{z}$ direction, a substantial complication, which is nevertheless worth pursuing in the future.

\section{III). Elements of Magnetohydrodynamics (MHD) Theory}

There are two basic levels of theoretical description: 1). Microscopic description, which was covered in reference 1, also referred to as kinetic theory, which is based on phase space distribution of plasma particles utilizes mainly the Vlasov and some of Maxwell's equations. One of the main virtues of this theory is the natural manner in which finitetemperature effects are included. 2).Macroscopic fluid description, to be covered next, is based on moments of the Liouville or the Boltzmann equations, some of Maxwell's equations, and Ohm's law. The choice of equations in either theory is based on the problem to be solved.

Before proceeding, it is important to define a plasma. The most common definition is that a plasma is a collection of charged particles whose statistical properties are determined by multiple Coulomb interactions. Mathematically, it can be expressed as $n \lambda_{D} \gg 1$, where $\lambda_{D}$ is the Debye length. In essence, there should be many particles in a Debye sphere to have plasma collective effects.

Like any many-body system, a collection of charged particles can be described by a chain of statistical equations, which could or could not be closed. However, in a plasma, 
where $n \lambda_{D} \gg>1$ a statistical chain of Liouville equations can be simplified by neglecting terms of order $1 / n \lambda_{D}$. But, in cases where there are collisions, a collision integral must be added. And, once its form is established, the resulting transport equation is the Boltzmann equation,[3]

$$
\left[\frac{\partial}{\partial t}+v \cdot \nabla+\frac{q_{j}}{m_{j}}(E+v \times B) \cdot \nabla_{v}\right] f_{j}(r, v, t)=\left(\frac{\partial f}{\partial t}\right)_{\text {coll }}
$$

Taking the moments of the Boltzmann equation, yield the continuity, momentum transfer and energy transport equations for the $\mathrm{j}^{\text {th }}$ species, [4]

$$
\begin{gathered}
\frac{D^{j} n_{j}}{D t}+n_{j} \nabla \cdot \mathbf{v}_{\mathbf{j}}=0 ; \\
m_{j} n_{j} \frac{D^{j} \mathbf{v}_{\mathbf{j}}}{D t}=-\nabla p_{\mathrm{j}}-\nabla \cdot \mathbf{P}_{\mathbf{j}}+q_{j} n_{j}\left[\mathbf{E}+\mathbf{v}_{\mathbf{j}} \times \mathbf{B}\right]+\mathbf{F}_{\mathbf{j}} \\
\frac{3}{2} n_{j} k \frac{D^{j} T_{j}}{D t}+p_{j} \nabla \cdot \mathbf{v}_{\mathrm{j}}=-\nabla \cdot \mathbf{Q}_{\mathbf{j}}-\mathbf{P}_{\mathrm{j}}: \nabla \mathbf{v}_{\mathrm{j}}+{ }^{\prime} \mathrm{E}_{j}
\end{gathered}
$$

Here $D^{j} / D t=\partial / \partial t+v_{j} \cdot \nabla ; p_{j}=n_{j} k T_{j}$, where $k$ is Boltzmann's constant;

$\mathbf{P}$ is the stress tensor and $\mathbf{Q}$ is heat flow. $\mathbf{F}$ and $\mathbf{E}$ and momentum and energy gained.

Unlike atomic physics where the Schroedinger equation can be used to define and become the basis for solving a problem, plasma physics does not have a master equation. 
Instead, a set of equations must be chosen based on best guess as to which equations are most relevant. Usually, to close this set of equations, some of Maxwell's equations and Ohm's law, are added to equations 5 .

IV). MHD Modes

To solve for MHD modes, equations 5, some of Maxwell's equations, and Ohm's law are linearized and solved to obtain a dispersion relation. A dispersion relation for electrostatic oscillations in a nonneutral plasma similar to an EBIS has been derived. [5]

$$
\begin{gathered}
k_{z} R_{p} \frac{K_{n}\left(k_{z} R_{c}\right) I_{n}^{\prime}\left(k_{z} R_{p}\right)-K_{n}^{\prime}\left(k_{z} R_{p}\right) I_{n}\left(k_{z} R_{c}\right)}{K_{n}\left(k_{z} R_{c}\right) I_{n}\left(k_{z} R_{p}\right)-K_{n}\left(k_{z} R_{p}\right) I_{n}\left(k_{z} R_{c}\right)} \\
-\left(1-\sum_{j} \frac{\bar{\omega}_{p j}^{2}}{v_{j}^{2}} T_{p} \frac{J_{n}^{\prime}\left(T R_{p}\right)}{J_{n}\left(T R_{p}\right)}=n \sum \frac{\bar{\omega}_{p j}^{2}\left(n \Omega_{j}+2 \omega_{j}\right)}{v_{j}^{2}\left(\omega-k_{z} V_{j z}^{0}-n \omega_{j}\right)}\right. \\
T^{2} \equiv-k_{z}^{2} \frac{\left[1-\sum_{j} \frac{\bar{\omega}_{p j}^{2}}{\left(\omega-k_{z} V_{z \mathrm{j}}^{0}-\ell \omega_{j}\right)^{2}}\right]}{\left[1-\sum_{j} \frac{\bar{\omega}_{p j}^{2}}{v_{j}^{2}}\right]} \\
\omega_{e}=\omega_{e}^{ \pm}=\frac{\Omega_{e}}{2}\left\{1 \pm\left[1-\frac{2 \bar{\omega}_{p e}^{2}}{\Omega_{e}^{2}}(1-\phi)\right.\right. \\
\omega_{i}=\omega_{i}^{ \pm}=\frac{\Omega_{i}}{2}\left\{1 \pm\left[1+\frac{2 \bar{\omega}_{p i}^{2}}{\Omega_{i}^{2}} \frac{1-\phi}{\phi}\right]^{1 / 2}\right\}, \\
\text { and } \\
v_{j}^{2}=\left(\omega-k_{z} V_{j z}^{0}-\omega_{j}\right)^{2}-\left(n \Omega_{j}+2 \omega_{j}\right)^{2} .
\end{gathered}
$$


$R_{p}$ and $R_{c}$ are the plasma and conductor radii respectively. The "prime" notation denotes derivatives with respect to the complete argument of the Bessel function. This equation is a fairly rigorous dispersion relation for electrostatic oscillations in a cold, constant density, plasma. Equation 6 cannot be solved to obtain a close analytical expression for $\omega$ unless simplifying approximations are made. Hence, analysis can be made for a few limiting cases only. Since no violent MHD modes are expected in an EBIS, i.e., no large radial displacements, EBIS researchers need to concentrate on surface waves and azimuthal rotational modes like various rotating stream instabilities, and various diocotron instabilities. Elements of mode analysis $[6,7,8,9]$ reveal that the various modes are driven by azimuthal rotations and beam current. Both of these free energy sources do exist in EBIS. Some of the modes to watch for are: rotating stream instabilities [8], diocotron and resonant diocotron, [9] and beam-plasma. [6,10] It should be noted that the analysis involves a long wavelength approximation. As such, these modes could not occur in an EBIT, and the analysis is valid only in an EBIS with $R_{p}>>\lambda_{D}$.

\section{V). Discussion}

Catastrophic MHD modes (like kink and sausage) cannot occur in EBIS devices due to the relatively low current and very high magnetic field. But, in an EBIS where $R_{p}>>\lambda_{D}$ and $k_{2} R_{p}<<1$ are satisfied, rotating stream instabilities, diocotron and resonant diocotron instabilities, and beam-plasma instabilities can occur providing the necessary conditions are satisfies as well. A sufficient condition for stability against diocotron modes, is for the spatial distribution function to be monotonically decreasing.[9] A halo resulting from stripped electrons will violate this condition. By its nature, an EBIS contains the free energy sources 
needed for electron-ion two-rotating-stream, and beam-plasma instabilities. Next, suggestions are made for instability prevention (in case one is encountered).

Feedback stabilization should be effective for surface waves, like diocotron modes. Preventing these modes from occurring can be effectively done by sweeping away the stripped electrons. Adding an additional pair of drift tubes, which generate an electric field component, that guides the stripped slow electron to a collection plate, will prevent any diocotron mode from occurring.

Ion cooling can reduce the potency of the electron-ion two-rotating-stream instability. Cooling the ions, forces the ions to spend more time in the electron beam, makes the column more uniform, prevents formation of contrarotating annuli or cylinders. Another possibility for controlling ion rotation is with a rotating field drive, in which a sinusoidal field is applied to a segmented drift tube (it may also enhance overall confinement by maintaining the constancy of the canonical angular momentum).

The beam-plasma instability or the Buneman instability should not generally be a problem in an EBIS. But, should such an instability be encountered, the $R_{p} / R_{c}$ ratio must be increased to raise the current threshold.[5,6]

As a last resort, broad band high frequency noise was shown to be effective in stabilizing lower frequency instabilities [11], and in reducing transport losses [12,13].

Nonlinear effects and well as physics at the trap gates, electron gun and collector was not addressed in this paper. Rotational frequency variations in the $z$ direction at the trap edges can create shear and oscillations. Due to communication along the electron beam, oscillation in a collector, e.g., can trigger instabilities in the EBIS trap. 


\section{References}

1). A. Hershcovitch, Physica Scripta, T71, 34 (1996).

2). B.D. Fried and S.C. Conte, The Plasma Dispersion Function, Academic Press Inc., New York, 1961.

3). L.D. Landau, and E.M. Lifshitz, Statistical Mechanics, Pergamon Press London, 1958.

4). Derivation can be found in many text books, e.g, L. Spitzer, Physics of Fully Ionized Gases, John Wiley and Sons, Inc., New York, 1962.

5). L.M. Linson, Phys. Fluids, 14, 805 (1971); see also R. Davidson Theory of Nonneutral Plasmas, W.A. Benjamin, Inc., Advanced Book Program, Reading MA 1974.

6). I.B. Bernstein and S.K. Trehan, Nucl. Fusion 1, 3 (1960).

7). R.H. Levy, Phys. Fluids, 8, 1288 (1965).

8). R.H. Levy, J.D. Daugherty, and O. Buneman, Phys. Fluids, 12, 2616 (1969).

9). R.J. Briggs, J.D. Daugherty, R.H. Levy, Phys. Fluids, 13, 421, (1970).

10). L.S. Bogdanskevich and A.A. Rukhadze, Sov. Phys.-Usp. 14, 163 (1971).

11). A. Hershcovitch, and P. Politzer, Phys. Fluids, 22, 249 (1979).

12). A. Hershcovitch, and P. Politzer, Phys. Fluids, 22, 1497 (1979).

13). A. Hershcovitch, and P. Politzer, Phys. Fluids, 25, 522 (1982). 